\title{
Impacto da multimistura no estado nutricional de pré-escolares matriculados em creches
}

\author{
Impact of a dietary supplement on the nutritional \\ status of preschool children enrolled in day care centers
}

Susy Mary Souto de OLIVEIRA ${ }^{1}$

Maria José de Carvalho COSTA ${ }^{1}$

Maria Amélia Amado RIVERA ${ }^{2,3}$

Leonor Maria Pacheco SANTOS 4

Maria de Lourdes Coelho RIBEIRO ${ }^{5}$

Geórgia de Sousa Ferreira SOARES 6

Luisa Sônia ASCIUTTI ${ }^{1}$

Solange Fátima Geraldo da $\operatorname{COSTA}^{7}$

RE S U M O

\section{Objetivo}

Avaliar o impacto da suplementação da dieta com multimistura sobre o estado nutricional de crianças em fase pré-escolar em risco nutricional.

\section{Métodos}

Trata-se de estudo tipo duplo cego com duração de dois meses e acompanhamento longitudinal de 135 crianças na faixa etária de um a seis anos. As crianças foram divididas em três grupos: intervenção $1(G \mid 1 n=48)$, intervenção $2(G \mid 2 n=45)$ e controle $(G C n=42)$, recebendo $5 \mathrm{~g}$ e $10 \mathrm{~g}$ de multimistura e placebo, respectivamente. O estado nutricional das crianças em estudo foi avaliado antes e após a suplementação.

\footnotetext{
1 Programa de Pós-Graduação em Ciências da Nutrição, Centro de Ciências da Saúde, Universidade Federal da Paraíba. João Pessoa, PB, Brasil.

2 Departamento de Nutrição, Centro da Saúde, Faculdade de Ciências Médicas da Paraíba. Praça Dom Ulrico, 56, Centro, 58010-740, João Pessoa, PB, Brasil. Correspondência para/Correspondence to: S.M.S. OLIVEIRA. E-mail: <susysouto@terra.com.br>.

3 Núcleo Interdisciplinar de Estudos em Saúde e Nutrição, Universidade Federal da Paraíba. João Pessoa, PB, Brasil.

4 Departamento de Nutrição, Universidade de Brasília. Brasília, DF, Brasil.

5 Discente, Curso de Nutrição, Centro da Saúde, Faculdade de Ciências Médicas da Paraíba. João Pessoa, PB, Brasil.

6 Discente, Curso de Nutrição, Centro de Ciências da Saúde, Universidade Federal de Paraíba. João Pessoa, PB, Brasil.

7 Programa de Pós-Graduação em Enfermagem, Centro de Ciências da Saúde, Universidade Federal de Paraíba. João Pessoa, PB, Brasil.
} 
170 | S.M.S. OLIVEIRA et al.

\section{Resultado}

Não houve diferença significativa nos indicadores peso/idade, altura/idade e peso/altura entre os grupos estudados na linha base $(p=0,251 p=0,248$ e $p=0,399$, respectivamente). Após a intervenção a diferença na prevalência de déficit antropométrico entre os grupos também não foi significante $(p=0,100 p=0,435$ e $p=0,210$, respectivamente). A comparação das médias antes e depois da intervenção, igualmente, não demonstrou diferenças ( $p>0,05$ utilizando os métodos estatísticos de Kruskall - Wallis e Análise de Variância).

\section{Conclusão}

Frente ao impacto das pequenas quantidades consumidas de suplemento, no caso a mistura de farelo de cereais, não se justifica esperar alterações significativas sobre a recuperação de crianças em risco nutricional, já que esses suplementos contêm somente pequenas quantidades de calorias e de macro e micronutrientes.

Termos de indexação: estado nutricional; multimistura; suplementação alimentar.

\section{A B S T R A C T}

\section{Objective}

The objective of this study was to evaluate the impact of a diet supplemented with a bran-based cereal mixture on the nutritional status of preschool children presenting nutritional risk.

\section{Methods}

This was a two-month double-blinded study with a longitudinal follow up using 135 children with ages ranging from one to six years old and divided into three groups: intervention $1(G / 1=48)$, intervention $2(G / 2$ $n=45)$ and control (CG $n=42)$, receiving $5 g$ and $10 \mathrm{~g}$ of the multi-mixture and placebo, respectively. The nutritional situation of the children in the study was evaluated before and after supplementation.

\section{Results}

No significant differences were observed in the weight/age, height/age and weight/height indicators at the base line $(p=0.251 p=0.248$ and $p=0.399$ respectively) between the groups studied. After intervention, the difference between the groups in the prevalence of anthropometrical deficit was also not significant $(p=0.100$ $p=0.435$ and $p=0.210$ respectively) and a comparison of the averages before and after intervention revealed no differences ( $p>0.05$ using the Kruskall - Wallis and Analysis of Variance statistical methods).

\section{Conclusion}

In the specific case of the bran-based mixture, the impact of the small amounts of supplement ingested did not warrant expecting alterations in the recovery of children presenting nutritional risk, since these supplements contain only small amounts of calories and macro and micronutrients.

Indexing terms: nutritional status; nutritional supplement; supplementary feeding.

\section{N T R O D U Ç Ã O}

Apesar do progresso verificado nos últimos 15 anos nos países em desenvolvimento, aproximadamente $40,0 \%$ das crianças com menos de cinco anos de idade (cerca de 200 milhões de crianças) são mal nutridas, quando avaliadas pelo déficit estatural ${ }^{1}$. Entre as crianças pré-escolares brasileiras, segundo o indicador altura para idade, a prevalência da desnutrição é de 10,5\%, sendo ainda mais acentuada na região Nordeste $(17,9 \%)^{2}$. Na Paraíba, no projeto "Criança contra a fome e pela vida" - subprojeto Paraíba, no ano de 2000, foi realizado um diagnóstico antropométrico em 87229 crianças, apontando um grave quadro de desnutrição aguda, cuja prevalência média foi de 43,3\% nos 86 municípios atendidos pelos Agentes Comunitários de Saúde 3 .

A década de 90 , do século $X X$, assinala um evento histórico no que se refere ao tratamento dos problemas nutricionais que foram discutidos na Conferência de Cúpula de Nova lorque, que estabeleceu o compromisso internacional de inscrever a problemática nutricional no quadro de prioridades de saúde coletiva até o ano 
2000. Reconhecia-se, com essa decisão, a importância dos problemas carenciais e, muito especialmente, da desnutrição energético-protéica (DEP) e/ou protéico-calórica (DPC) em crianças, em face de sua peculiar vulnerabilidade biológica às deficiências nutricionais ${ }^{4}$.

Os países em desenvolvimento têm aplicado, como estratégia para o combate à desnutrição, suplementos alimentares que utilizam desde produtos altamente energéticos, como os biscoitos que fornecem $1000 \mathrm{cal} / \mathrm{dia}^{5}$, até produtos de valor intermediário e de pequeno valor energético. Desse modo, várias alternativas estão sendo desenvolvidas com o objetivo de reduzir a prevalência da desnutrição; no Brasil, uma das estratégias das entidades filantrópicas e públicas para amenizar o problema da desnutrição é a implementação de projetos de suplementação alimentar, dentre os quais a distribuição de misturas à base de farelo de cereais, popularmente denominadas de multimisturas ${ }^{6,7}$.

A utilização e os padrões de identidade e de qualidade da multimistura foram regulamentados pela Agência Nacional de Vigilância Sanitária (ANVISA/MS) a partir da resolução n53, que definiu a mistura à base de farelo de cereais como sendo "produto obtido pela secagem, torrefação, moagem e mistura de ingredientes de origem vegetal, podendo ser adicionada de leite em pó" 8 .

A eficácia desse suplemento, no entanto, é objeto de controvérsias. Segundo Farfan , não há provas de que esse produto seja adequado para garantir manutenção e crescimento à população infantil por longos períodos, considerando que seu valor nutricional como complemento, sua composição e as propriedades biológicas dos seus ingredientes não são condizentes com as propriedades especiais que seus preconizadores defendem. A multimistura poderia, provisoriamente, evitar o óbito por inanição, em casos de extrema pobreza e absoluta falta de alimentos ${ }^{7}$.

A produção da multimistura é realizada em diversos setores e serviços e em diversas escalas, até mesmo artesanalmente, em ambiente domi- ciliar. Na Paraíba, o Hospital Universitário Lauro Wanderley, por meio do setor de puericultura, também produz a multimistura e a distribui entre as crianças que freqüentam o hospital e seu ambulatório. Cada $100 \mathrm{~g}$ daquela preparação contém $47,5 \%$ de farelo de trigo, $47,5 \%$ de fubá de milho, $4,0 \%$ de pó de semente de melão, gergelim, abóbora e amendoim, 0,5\% de pó de folha de mandioca e $0,5 \%$ de casca de ovo, e apresenta a seguinte composição centesimal: $2,7 \mathrm{~g}$ de cinzas, 5,2g de lipídios, $11,7 \mathrm{~g}$ de proteínas, $74,2 \mathrm{~g}$ de carboidratos e 6,2g de fibras. Em sua composição mineral, apresenta, em $100 \mathrm{~g}$ do produto, $8 \mathrm{mg}$ de ferro, $357 \mathrm{mg}$ de cálcio, $235 \mathrm{mg}$ de magnésio, $677 \mathrm{mg}$ de potássio, 570mg de fósforo e $7 \mathrm{mg}$ de sódio 9 .

Considerando o uso rotineiro do produto como forma de combate à desnutrição, fica evidenciada a necessidade de um estudo cujo objetivo seja avaliar o impacto da suplementação com mistura de farelo à base de cereais no controle da desnutrição protéico-energética, adicionada à dieta habitual de crianças em idade pré-escolar em creches municipais de João Pessoa.

\section{MÉ TOD OS}

Este trabalho trata de um estudo de acompanhamento longitudinal, tipo duplo cego, com duração de dois meses, tempo suficiente, segundo Lesourd et al. ${ }^{10}$, para serem observadas alterações nutricionais.

O estudo foi realizado em quatro creches municipais de João Pessoa, sorteadas aleatoriamente, de um total de 15, correspondendo, portanto, a 27,0\% do total. Elas assistem 1200 crianças na faixa etária de um a seis anos, e situam-se, geralmente, em áreas carentes, onde $57,4 \%$ das famílias percebem uma renda familiar entre um e dois salários mínimos. As creches sorteadas atendem 250 crianças, correspondendo, esse número, a 21,0\% do total de crianças atendidas em todas as creches municipais. Essa quantidade foi reduzida para 135 crianças devido aos critérios de exclusão utilizado neste estudo, 
quais sejam: estar em uso de medicamentos como sulfato ferroso, suplementos vitamínicos ou outro medicamento $(n=22)$, ausentar-se da creche durante seis dias $(10,0 \%$ do tempo total do estudo, n=90) no período da suplementação, ou apresentar adequação de peso para a idade <-3 Z-escores $(n=3)$. Os critérios de exclusão levaram em conta as questões éticas, principalmente, resultados de trabalhos científicos que questionam um comprometimento imunológico e metabólico devido à alta ingestão de fibras e grãos integrais ${ }^{11}$.

O cálculo do tamanho da amostra, realizado no programa Epi Info' ${ }^{12}$, indicou que, incluindo-se 41 crianças no grupo-controle e 82 no grupo intervenção, a amostra teria um poder de $80,0 \%$ para detectar uma alteração na prevalência de desnutrição de $25,0 \%$ para 5,0\%, considerando um limite de confiança de 95,0\%.

As crianças de cada creche foram distribuídas em três grupos: dois grupos de intervenção (GI1, composto por 48 crianças e GI2, por 45 crianças) e um grupo-controle (GC) com 42 crianças.

O GC recebeu $5 \mathrm{~g}$ de placebo (farinha de mandioca), semelhante à mistura de farelo de cereais em coloração e espessura dos grãos, apresentando, em $100 \mathrm{~g}$ do produto, a seguinte composição centesimal13: 336,8 calorias, $81,1 \mathrm{~g}$ de carboidratos, 2,2g de proteínas, $0,05 \mathrm{~g}$ de lipídios, $21 \mathrm{mg}$ de cálcio, $105 \mathrm{mg}$ de fósforo e $0,8 \mathrm{mg}$ de ferro. O Gl1 recebeu $5 \mathrm{~g}$ da mistura de farelo à base de cereais (procedimento adotado pelo Hospital Universitário Lauro Wanderley) e o GI2 recebeu $10 \mathrm{~g}$ da mistura de farelo de cereais, para comparar melhor os resultados obtidos com os estudos experimentais anteriores, que utilizaram doses diferentes da multimistura ${ }^{9,14}$. Nenhuma alteração no esquema alimentar foi proposta às creches.

Para a participação do menor no projeto, foi solicitado o consentimento livre e esclarecido dos pais, com quem foram discutidos os objetivos do projeto e os procedimentos a serem realizados. O protocolo de pesquisa foi aprovado pelo Comitê de Ética do Hospital Universitário Lauro Wanderley, da Universidade Federal da Paraíba.

A ingestão alimentar foi avaliada utilizando-se o método resto/ingestão durante três dias, antes e após a suplementação, visando avaliar a porção consumida. As preparações oferecidas (desjejum, almoço, lanche e jantar) foram identificadas quanto aos seus componentes e per capita ${ }^{15,16}$. Os pratos foram marcados com etiquetas contendo os respectivos nomes das crianças, com codificação. Após a identificação dos pratos, era misturado à alimentação, o placebo ( $5 \mathrm{~g}$ da farinha de mandioca) para as crianças do GC e a multimistura, para os grupos experimentais (Gl1 - $5 \mathrm{~g}$ da multimistura e GI2 - 10g da multimistura), sempre por um único membro da equipe, especialmente treinado.

As substâncias adicionadas à dieta das crianças eram misturadas ao feijão e esse, em seguida, misturado ao restante dos alimentos, de forma bastante homogênea. Os alimentos foram medidos e/ou pesados para controle do consumo e das sobras das refeições servidas, durante os três dias do estudo.

Para o controle da pesagem dos alimentos sólidos e pastosos, utilizou-se uma balança dietética marca Bender semi-analítica, com capacidade para $10 \mathrm{~kg}$. Para os alimentos líquidos foi utilizado um copo medida graduado de $500 \mathrm{ml}$ e escala de $25 \mathrm{ml}$, ou a própria mamadeira. Após o término das refeições, os pratos eram examinados para a obtenção do volume de ingestão de cada criança. A partir desse procedimento, foi calculada a ingestão de calorias. Considerando que a quantidade de substâncias adicionadas ao alimento era muito pequena, não houve alteração considerável no teor energético nem no sabor da dieta, não havendo, portanto, interferência na aceitação pelas crianças. As sobras dos pratos eram desprezíveis, pois já era hábito das crianças consumirem toda a porção de alimento oferecida.

A média de calorias fornecida pela multimistura era de 18,4 para GI1 , 36,8 para GI2 e 16,8 para GC, o que corresponde, aproximadamente, a $1,6 \%$ da energia total consumida, 
por dia, no Gl1 (1 144 calorias), 3\% no GI2 (1 196 calorias) e 1,5\% no GC (1 194 calorias).

As crianças foram submetidas à avaliação antropométrica no início e no final do estudo, seguindo as recomendações do Ministério da Saúde ${ }^{17}$. As maiores de dois anos foram pesadas em balança marca Filizola antropométrica com o mínimo de vestimentas e sempre no período da manhã. A altura foi aferida utilizando-se o estadiômetro da própria balança. Os menores de dois anos foram pesados em balança pediátrica, mecânica, apoiada sobre uma superfície plana, lisa e firme. O comprimento foi medido por um antropômetro horizontal, apoiado em superfície plana, firme e lisa ${ }^{17}$. O padrão de referência utilizado foi o National Center of Health Statistic $(\mathrm{NCHS})^{18}$.

Foi utilizado um ponto de corte ${ }^{19}$ mais sensível para detectar alterações do estado nutricional; a normalidade nutricional foi atribuída às crianças com peso compreendido entre $+1,00$ e -1,00 Z-escores; o risco de desnutrição, com medidas entre -2,00 e -1,00 Z-escores; desnutrição moderada entre $-3,0$ e $-2,01$ e excesso de peso com medidas maiores que $+1,00$ Z-escores. Para calcular os índices antropométricos, utilizou-se o módulo Epinut do programa Epi Info 6.04². Para avaliar a homogeneidade dos grupos no início do estudo e analisar a eficácia da intervenção, empregou-se a comparação de médias por meio do teste $t$ (Anova), quando apropriado, ou do teste não paramétrico Kruskal-Wallis, quando as variâncias entre os grupos diferiram significantemente. O consumo de alimentos foi avaliado pelo software CIS-EPM versão $2.4^{20}$.

\section{RES ULTADOS}

Na Tabela 1, estão representados os dados do estado nutricional das crianças estudadas, antes e após a suplementação, de acordo com os três indicadores expressos em Escores Z (P/l, A/l e P/A). Verifica-se que a maioria das crianças de ambos os grupos continou na faixa de normalidade (entre $-1,00$ e +1,00 Z-score), enquanto 10,0\% a $20,0 \%$ das crianças dos GI1 e GI2 e do GC apresentaram-se com excesso de peso e permaneceram nessa condição. Entretanto, 6,3\% do Gl1, $4,4 \%$ do GI2 e $2,4 \%$ do GC estavam com déficit

Tabela 1. Distribuição das crianças das creches municipais de João Pessoa, PB, antes e após a suplementação, segundo indicadores antropométricos

\begin{tabular}{|c|c|c|c|c|c|c|c|}
\hline \multirow{3}{*}{$\begin{array}{l}\text { Indicador nutricional } \\
\text { (z-escore) }\end{array}$} & \multirow{3}{*}{ Grupo } & \multicolumn{6}{|c|}{ Z-escore } \\
\hline & & \multicolumn{2}{|c|}{ Peso/Idade } & \multicolumn{2}{|c|}{ Altura/Idade* } & \multicolumn{2}{|c|}{ Peso/Altura* } \\
\hline & & Antes $^{1}$ & Após ${ }^{2}$ & Antes $^{3}$ & Após ${ }^{4}$ & Antes $^{5}$ & Após $^{6}$ \\
\hline \multirow[t]{3}{*}{$>+1,00$} & GI1 & 6,3 & 4,2 & 10,4 & 4,2 & 10,4 & 16,7 \\
\hline & GI2 & 13,3 & 11,1 & 6,7 & 2,2 & 15,6 & 13,3 \\
\hline & $\mathrm{GC}$ & 19,0 & 19,0 & 4,8 & 4,8 & 21,4 & 28,6 \\
\hline \multirow[t]{3}{*}{$-1,00>$ e $<+1,00$} & GI1 & 77,1 & 79,2 & 68,8 & 70,8 & 77,1 & 75,0 \\
\hline & GI2 & 60,0 & 60,0 & 73,3 & 75,6 & 66,7 & 71,1 \\
\hline & $\mathrm{GC}$ & 61,9 & 61,9 & 57,1 & 57,1 & 71,4 & 66,7 \\
\hline \multirow[t]{3}{*}{$-2,00>$ e $<-1,01$} & GI1 & 16,7 & 16,7 & 14,6 & 20,8 & 8,3 & 8,3 \\
\hline & GI2 & 26,7 & 28,9 & 15,6 & 17,8 & 15,6 & 13,3 \\
\hline & $\mathrm{GC}$ & 19,0 & 19,0 & 35,7 & 35,7 & 7,1 & 4,8 \\
\hline \multirow[t]{3}{*}{$-3,00>$ e $<-2,01$} & GI1 & 0 & 0 & 6,3 & 4,2 & 4,2 & 0 \\
\hline & $\mathrm{G} 12$ & 0 & 0 & 4,4 & 4,4 & 2,2 & 2,2 \\
\hline & $\mathrm{GC}$ & 0 & 0 & 2,4 & 2,4 & 0 & 0 \\
\hline
\end{tabular}

Teste $\chi^{2}$ Pearson; ${ }^{1} p=0,251 ;{ }^{2} p=0,100 ;{ }^{3} p=0,248 ;{ }^{4} p=0,435 ;{ }^{5} p=0,399 ;{ }^{6} p=0,210$; Grupo intervenção 1 (n=48); Grupo intervenção 2 (n=45) e Grupo controle $(n=42)$

Fonte: Dados coletados em crianças das creches municipais de João Pessoa, PB,1999. 
de altura para a idade antes da intervenção, quadro que permaneceu quase inalterado depois dela. As diferenças de proporção, quando testadas com o $\chi^{2}$ de Pearson, não se mostraram significativas $(p>0,05)$ (Tabela 1).

De modo geral, dentro de cada grupo, as médias dos indicadores $\mathrm{P} / \mathrm{l}$ e $\mathrm{A} / \mathrm{l}$ foram menores, enquanto o inverso ocorreu em relação ao indicador P/A após a intervenção (Tabela 2). Vale salientar que a alocação das crianças por grupo foi aleatória (por sorteio) e, embora não significativas, as diferenças observadas explicam-se simplesmente pela chance. Contudo, deve-se observar que não houve diferença estatística entre os grupos, antes e após a intervenção.

\section{I S C U S S Ã O}

A necessidade de melhorar o estado nutricional da população de baixo poder aquisitivo tem levado diversos segmentos da sociedade organizada, no Brasil, a buscar alternativas para minimizar carências nutricionais existentes por meio de produtos alimentares não convencionais, como a mistura à base de farelo de cereais ${ }^{7,21}$.

A situação socioeconômica das famílias da maioria das crianças deste estudo é semelhante à situação de uma grande parte da população brasileira, ou seja, orçamento familiar em torno de um a dois salários mínimos.
Ao avaliar o impacto do consumo da multimistura no estado nutricional das crianças, não foram detectadas diferenças na alteração da média do peso e/ou da altura entre os grupos, tanto no momento inicial do estudo quanto no momento final. Dentro de cada grupo, as médias dos indicadores $\mathrm{P} / \mathrm{I}$ e $\mathrm{A} / \mathrm{I}$ foram discretamente menores após a intervenção, o que pode indicar uma deterioração do estado nutricional, mas ocorreu o inverso em relação ao indicador P/A, a partir do qual se observou uma discreta melhora. Não se pode, no entanto, afirmar que as sutis alterações foram devidas à suplementação, uma vez que as diferenças observadas no estado nutricional, após a intervenção, não foram significantes.

Tais resultados estão de acordo com dados de outras pesquisas que avaliaram o impacto do consumo da multimistura e outros alimentos em crianças ${ }^{21,22}$, e também com resultados de estudos experimentais, com ratos ${ }^{14,23,24}$. Diante dos anseios da população que faz uso da multimistura e da ênfase dada por alguns profissionais de nível superior aos efeitos da mesma, poder-se-ia esperar que as crianças deste estudo melhorassem seu estado nutricional. É preciso ressaltar que foram demonstrados resultados positivos quanto ao uso da multimistura, contrários àqueles mencionados acima, em estudos anteriores ${ }^{25,26}$, dos quais se podem questionar alguns aspectos metodológicos fundamentais:

Tabela 2. Estado nutricional das crianças das creches municipais de João Pessoa, PB, antes e após suplementação, segundo os indicadores antropométricos.

\begin{tabular}{|c|c|c|c|c|c|c|c|c|c|}
\hline \multirow{2}{*}{ Variável } & \multirow{2}{*}{ Grupos } & \multicolumn{2}{|c|}{ Mediana } & \multicolumn{2}{|c|}{ Média } & \multicolumn{2}{|c|}{ Desvio-padrão } & \multicolumn{2}{|c|}{ Teste estatístico } \\
\hline & & Antes & Após & Antes & Após & Antes & Após & Antes & Após \\
\hline \multirow{3}{*}{$\begin{array}{l}\text { Peso/Idade } \\
\text { (Z-escore) }\end{array}$} & GI1 & $-0,120$ & $-0,115$ & $-0,208$ & $-0,238$ & 0,84 & 0,82 & $F=0,16$ & $F=0,35$ \\
\hline & GI2 & $-0,160$ & $-0,330$ & $-0,278$ & $-0,239$ & 1,11 & 1,03 & $p=0,850$ & $p=0,707$ \\
\hline & GC & 0,530 & $-0,450$ & 0,153 & $-0,083$ & 1,12 & 1,07 & & \\
\hline \multirow{3}{*}{$\begin{array}{l}\text { Altura/ldade } \\
\text { (Z-escore) }\end{array}$} & GI1 & $-0,280$ & $-0,390$ & $-0,255$ & $-0,401$ & 1,10 & 0,98 & $F=2,13$ & $F=1,28$ \\
\hline & $\mathrm{G} / 2$ & $-0,300$ & $-0,320$ & $-0,341$ & $-0,487$ & 0,90 & 0,85 & $p=0,112$ & $p=0,252$ \\
\hline & GC & $-0,680$ & $-0,730$ & $-0,677$ & $-0,721$ & 0,97 & 0,95 & & \\
\hline \multirow{3}{*}{$\begin{array}{l}\text { Peso/Altura } \\
\text { (Z-escore) }\end{array}$} & GI1 & 0,030 & 0,175 & $-0,064$ & 0,109 & 0,84 & 0,80 & $F=2,47$ & $F=2,10$ \\
\hline & $\mathrm{G} / 2$ & $-0,010$ & 0,170 & $-0,031$ & 0,099 & 1,13 & 1,08 & $p=0,291$ & $p=0,350$ \\
\hline & GC & 0,290 & 0,250 & 0,310 & 0,426 & 1,03 & 1,03 & & \\
\hline
\end{tabular}

GI1 Grupo intervenção 1 ( $n=48) ; G \mid 2=$ Grupo intervenção 2 ( $n=45) ; G C=$ Grupo controle $(n=42)$.

Fonte: Crianças das creches municipais de João Pessoa, PB, 1999. 
- Falta de controle de fatores de confundimento, tais como programas educativos, incentivo ao aleitamento materno, vacinação em dia, melhor aproveitamento dos alimentos, maiores cuidados com a higiene alimentar e pessoal, programas de distribuição de leite, de pão, suplementos de vitaminas e ferro, que poderiam interferir no estado nutricional das crianças que consumiam, concomitantemente, a multimistura.

- Ausência de grupo-controle, pois somente a utilização de placebo e a realização de estudos do tipo duplo cego podem evitar tendenciosidade dos resultados.

- Falta de controle quanto à ingestão dos alimentos oferecidos nas creches e domicílios.

Além desses fatos, alguns autores acreditam na necessidade de estudos visando estabelecer melhor tecnologia de preparo, garantindo adequadas condições higiênico-sanitárias ao produto, evitando, assim, possíveis efeitos tóxicos a longo prazo 7,27 .

O padrão microbiológico da mistura de farelo de cereais produzida pelo Setor de Puericultura do Hospital Universitário Lauro Wanderley, da Universidade Federal da Paraíba, foi estudado e os autores concluíram que, apesar de os valores de atividade de água e umidade estarem abaixo dos valores estabelecidos para as farinhas, fécula e fubá, indicando um bom armazenamento, isso não impediu o desenvolvimento de fungos e leveduras ${ }^{28}$. As amostras apresentaram-se dentro dos padrões estabelecidos para alimentos semelhantes em relação aos bolores e leveduras; porém, deve-se levar em consideração que esse suplemento alimentar, contendo fungos produtores de micotoxinas, quando consumido por pessoas com sistema imunológico deprimido, pode acarretar toxinfecções. Em consideração a esse fato, fica assim evidente a necessidade de obedecer aos critérios microscópicos e microbiológicos recomendados pela resolução $n^{\circ} 53$, nos casos em que a mistura de farelo de cereais seja preparada em domicílio e outro local que não obedeça ao referido critério ${ }^{8}$.

Um fato preocupante registrado nesta pesquisa foi a presença de crianças apresentando excesso de peso e obesidade. Esse fato vem sendo observado em todo o país, caracterizando um momento de transição nutricional, ou seja, declínio das enfermidades carenciais e ascensão da obesidade, o que leva a um questionamento para a saúde pública a respeito dos princípios, na educação nutricional, que devem ser enfatizados para prevenir e/ou tratar essa enfermidade ${ }^{29}$.

Urge a necessidade de colocar em prática estratégias e ações que atenuem, efetivamente, os desvios nutricionais em crianças. A esse respeito, Peixinho ${ }^{30}$ faz uma reflexão, anunciando que "é preciso combater as formas paliativas de combate à fome, como consumo de farelo pela população de baixa renda". Pode-se também questionar os programas de alimentação infantil que se propõem a atender em torno de 15\% das necessidades nutricionais diárias, com $R \$ 0,16 /$ dia. É necessário desvincular esses programas, de falsos e inatingíveis objetivos, de se constituírem em solução para a desnutrição, e ter uma proposta concreta e viável para erradicar esse mal que atinge 32 milhões de brasileiros, com projetos eficientes, como programas de transferência de renda e a definição de uma renda mínima satisfatória para a manutenção da família.

\section{REFERÊ NCIAS}

1. United Nations Children's Fundation. Situação mundial da infância. Brasília: Unicef; 2000.

2. Brasil. Instituto Brasileiro de Geografia e Estatística. Pesquisa Nacional sobre Demografia e Saúde 1996. 2.ed. Rio de Janeiro: Bemfam; 1999.

3. Rivera MAA, Costa MJC, Cavalcanti APR. A nova nutrição. In: MAA Rivera, MJC Costa. Epidemiologia de problemas nutricionais: desnutrição infantil no Estado da Paraíba. João Pessoa: Editora Universitária; 2000. p.183-99.

4. United Nations Children's Fundation. World Summit for Children [Internet]. New York; 1990 [cited 2002 Apr 27]. Available from: http:// www.unicef.org/wsc/declare.htm

5. United Nations Children's Fundation. Situação mundial da infância. Brasília: Unicef; 1998.

6. Bittencourt SA. Uma alternativa para a política nutricional brasileira? Cad Saúde Pública. 1998; 14(3):629-36. 
176 | S.M.S. OLIVEIRA et al.

7. Farfan JA. Alimentação alternativa: análise crítica de uma proposta de intervenção nutricional. Cad Saúde Pública. 1998; 14(1):205-12.

8. Brasil. Secretaria Nacional de Vigilância Sanitária. Resolução RDC $n^{\circ}$ 53, de 15 de junho de 2000. Regulamento Técnico para Fixação de Identidade e Qualidade da Mistura a Base de Farelo de Cereais. Brasília: Ministério da Saúde; 2000. p.4.

9. Madruga MS, Santos HB, Bion FM, Antunes NLM. Avaliação nutricional de uma dieta suplementada com multimistura: estudo em ratos. Ciênc Tecnol Alim. 2004; 24(1):129-33.

10. Lesourd B, Pfitzenmeyer P, Ferry M, Bonnefoy M, Constants TLSM, et al. Augmentation des processus oxydatifs au demmarge des renutrition de patients ages atteints de malnutrition proteino - energetique. Essai de prevention terapheutique par une assossiation de micronutriments antioxydants [dissertation]. Ivry/ Siene, Françe: Unité de Medecine Nutritionelle Geriatrique; 2002.

11. Baxter IC. Paradoxos em fibras alimentares: biodisponibilidade de minerais. Nutri Res. 1999; 13(3):649-57.

12. Dean AG, Dean JA, Coulombier D, Brendel KA, Smith DC, Burton A, et al. Epi Info, version 6: a word processing database, and statistic program for epidemiology on microcomputers. Atlanta (Georgia): Centers of Disease Control Prevention; 1994.

13. Franco G. Tabela de composição química dos alimentos. 9.ed. São Paulo: Atheneu; 2004. p.307.

14. Bion FM, Pessoa DCNP, Lapa MAG, Campos FA, Antunes NLM, Lopes SML. Uso de uma multimistura como suplementação alimentar: Estudo com ratos. Arch Latinoam Nutr. 1997; 47(3): 242-7.

15. Pinheiro ABV, Lacerda EMA, Benzecry EH, Gomes MCS, Costa VM. Tabela para avaliação de consumo alimentar em medidas caseiras. 2.ed. Rio de Janeiro; 1994.

16. Teixeira $A B$, Luna NMM. Técnica dietética. Fator de correção em alimentos de origem animal e vegetal. Cuiabá: Universidade Federal do Mato Grosso; 1995.

17. Brasil. Ministério da Saúde. Vigilância alimentar e nutricional: Antropometria: como pesar e medir. Brasília: Ministério da Saúde; 2004.

18. World Health Organization. Physical status: the use and interpretation of anthopometry. Geneva; 1995. Technical Report Series, 854.

19. Organização das Nações Unidas para a Alimentação e Agricultura. Manejo de projetos comunitários de alimentação e nutrição: guia didático. Recife; 2000.
20. Anção MS, Cuppari L, Tudisco ES, Draibe SA, Sigulem D. CIS - EPM Programa de Apoio à Nutrição [Programa de Computador] versão 2.5. São Paulo: Escola Paulista de Medicina; 1995.

21. Coelho MASC, Curvoni CC, Coelho KSC. Avaliação nutricional em crianças assistidas em uma creche da zona sul do município do Rio de Janeiro. Resumos do I Congresso Latinoamericano de Nutrição Humana. Gramado; 1999. p.36.

22. Prates ACM. Estudo do impacto do farelo de trigo, pó de folha de mandioca e pó de casca de ovo em crianças com risco de desnutrição [dissertação]. Recife: Universidade Federal de Pernambuco; 1998.

23. Frazão MF, Costa FEC, Costa MJC, Silva JAN. Histologia do cólon de ratos suplementados com multimistura. In: Anais da Jornada de Iniciação Cientifica; 2000. Brasília: Reunião Anual da SBPC; 2000. Pôster n6656 - A49I.

24. Boaventura GT, Chiappini CCJ, Assis Fernandes NR, Oliveira EM. Avaliação da quantidade protéica de uma dieta estabelecida em Quissamã, Rio de Janeiro, adicionada ou não de multimistura e de pó de folha de mandioca. Rev Nutr. 2000; 13(3):201-9.

25. Tonial SR. Estudos bromatológicos, antropométricos, bibliográficos e pesquisa de campo de um programa de intervenção nutricional de Santarém, Pará [monografia]. São Leopoldo: Centro de Ciências Biomédicas, Universidade do Vale dos Sinos; 1984.

26. Shrimpton R. Uma avaliação do Programa de Atenção Primária em Saúde sendo realizado em Santarém, Pará /Brasil pela Sociedade de Estudos e Aproveitamento dos Recursos da Amazônia (SEARA). Brasília: Unicef; 1994.

27. Camara FS, Madruga MS. Conteúdos de ácido cianídrico, ácido fítico, tanino total e aflotoxina em uma preparação brasileira (Natal) de multimistura. Rev Nutr. 2001; 14(1)33-6.

28. Santos HB. Estudos pré-clinicos da biodisponibilidade mineral da dieta enriquecida com multimistura consumida por crianças numa creche do município de João Pessoa, PB [dissertação]. João Pessoa: Centro de Tecnologia, Universidade Federal da Paraíba; 2000.

29. Batista Filho M. Rissin A. A transição nutricional no Brasil: tendências regionais e temporais. Cad Saúde Pública. 2003; 19(1):181-91.

30. Peixinho A. Alimentação e nutrição: atualidade, realidade e perspectivas. Rev Cons Fed Nutr. 2001; $3: 5$.

Recebido em: 28/3/2005

Versão final reapresentada em: 29/8/2005 Aprovado em: 13/10/2005 